\title{
Heterosis studies in CMS based upland Cotton (Gossypium hirsutum L.) hybrids
}

\author{
D. Shashibhushan ${ }^{1^{*}}$ and U. G. Patel ${ }^{2}$ \\ ${ }^{1}$ Seed Research and Technology Centre, PJTSAU, Hyderabad, India \\ ${ }^{2}$ Agricultural Research Station, Surath, Gujarat, India \\ *Corresponding author
}

\begin{tabular}{|c|}
\hline \\
\hline $\begin{array}{l}\text { Cotton, CMS, SCA } \\
\text { effects, Standard } \\
\text { Heterosis and Seed } \\
\text { cotton yield. }\end{array}$ \\
\hline Article Info \\
\hline $\begin{array}{l}\text { Accepted: } \\
18 \text { August } 2019 \\
\text { Available Online: } \\
10 \text { September } 2019\end{array}$ \\
\hline
\end{tabular}

A study of heterosis over checks was carried out with 24 Gosypium hirsutum entries comprising of $14 \mathrm{~F}_{1}$ s produced by CMS method, 7 females and 2 males and 1 check were evaluated at three locations viz., Surat, Bharuch and Hansot. The experiment was laid out in a Randomized Complete Block design (RBD) with three replications. CMS based crosses heterosis over standard check ranged from -39.17 to 9.36 per cent. Three crosses viz., G(B) 20 x G.Cot.10, G(B) 20 x DHY-286-1 and LRK-516 x DHY-286-1 performed better for standard heterosis, where their SCA effects were also significantly higher. It was observed that hybrids showing high heterosis for seed cotton yield per plant also manifested heterotic effects for its contributing characters like number of monopodia per plant, number of sympodia per plant, number of bolls per plant, boll weight, number of seeds per boll and seed index.

\section{Introduction}

Cotton, the king of the fibre is also called White Gold. Since cultivable land is limited and the importance of food crops cannot be ignored, increased demand could achieved only through increased production per unit area. The increased productivity can be achieved by developing superior varieties/ hybrids through genetic improvement and by proper management practices. Thus, the situation offers immense scope for geneticists in general and cotton breeders in particular both at national and state level. To meet the challenges of increasing productivity, Gossypium hirsutum L. offers better scope for genetic improvement among the four cultivated species of cotton. Majority of cotton produced by $G$. hirsutum species is medium and long staple. This species has very high adaptability with rich diversity for yield and yield related characters. On account of its versatility, the area under cultivation has increased tremendously in most cotton growing countries of the world with no exception to India and has created an 
increased interest in research on cultivation of G. hirsutum species. India is pioneer country in commercial exploitation of heterosis in cotton by developing several interspecific and intraspecific, hybrids for general cultivation. These cover nearly 40 per cent of cotton growing area and contribute $40-45$ per cent to the national production (Anonymous, 200102). However, at present the hybrid cotton seed is being produced by cumbersome and laborious process of hand emasculation and pollination. Probably this single largest factor has affected its further expansion and its production is not within the means of average farmer. To overcome the high cost of hybrid cotton seed, use of male sterility (as in sorghum, pearl millet etc.) could be the only answer in eliminating labour intensive manual emasculation. Use of male sterile lines appears to be advantageous since the maintenance of male sterile population for seed production is easier and more over sterility source under reference is stable. Cytoplasmic nuclear interaction affects the petal size and anther number which can be used as markers in identifying the parental lines and for ascertaining genetic purity. Accordingly the present study was planned and executed with producing hybrids with CMS system and evaluated for heterosis.

\section{Materials and Methods}

The present investigation was conducted with three complete sets of 24 Gosypium hirsutum entries comprising of $14 \mathrm{~F}_{1} \mathrm{~s}, 7$ females and 2 males and 1 check were evaluated during Kharif 2002 at three locations viz., Surat, Bharuch and Hansot.

The experiment was laid out in a Randomized Complete Block design (RBD) with three replications. The parents and $\mathrm{F}_{1} \mathrm{~s}$ with standard checks were represented by a single row plot of 14 plants, placed at $120 \mathrm{~cm} \mathrm{x} 45 \mathrm{~cm}$. All the agronomical practices and plant protection measures were followed as and when required to raise a good crop of cotton. The seeds of these parents were obtained from Main Cotton Research Station, Surat. For obtaining the cross seeds, parents were grown at Main Cotton Research Station, Surat.

The 7 females and 2 males were crossed in $\mathrm{L} \mathrm{x}$ $\mathrm{T}$ mating design to obtain 14 crosses of conventional hybrids making it totally 14 crosses. All the $\mathrm{F}_{1} \mathrm{~s}$ and selfed seeds of parents were stored properly in thick paper bags for sowing in the next season at three locations.

\section{Results and Discussion}

The estimates of heterosis measured as per cent increase or decrease over standard check (standard heterosis) in individual environment and on pooled basis are presented in Table 1a to $1 \mathrm{~d}$. In Days to 50 per cent flowering standard heterosis ranged from -32.55 to 6.20 per cent, whereas 13 crosses showed significant and negative standard heterosis. The crosses viz., LRK 516 x G.Cot.10, PH 93 x G.Cot.10, G (B) 20 x G.Cot.10 and LRA 5166 x G.Cot.10 exhibited maximum values of standard heterosis.

For plant height the magnitude of heterosis ranged from -34.54 to 10.48 per cent over standard check. Seven crosses showed significant and negative standard heterosis.

The crosses viz., LRK 516 x G.Cot.10, LRK 516 x DHY 286-1 and LH $900 \times$ G.Cot.10 showed maximum values of standard heterosis. The standard heterosis ranged from -29.32 to 44.58 per cent in number of monopodia per plant. Eight hybrids showed significant and positive standard heterosis.

The crosses viz, G(B) 20 x G.Cot.10, G(B) 20 $x$ DHY 286-1, 76 IH 20 x G.Cot.10, G.Cot.100 x G.Cot.10 and LRK 516 x DHY 286-1 recorded maximum standard heterosis. For number of sympodia per plant, the 
standard heterosis varied from -27.75 to 12.03 per cent and none of the crosses showed significant superiority over the standard check in desirable direction. In number of bolls per plant, the standard heterosis varied from 21.38 to 15.55 per cent. Three crosses viz., G(B) 20 x G.Cot.10, G.Cot.100 x DHY 286-1 and PH 93 x DHY 286-1 showed significant and positive standard heterosis.

The heterosis over standard check varied from -7.53 to 29.59 per cent in boll weight ( $\mathrm{g}$ ). The crosses which showed significant and positive standard heterosis were nine.

The crosses viz., G.Cot.100 x G.Cot.10, G(B) 20 x DHY 286-1, LRK 516 x DHY 286-1, LH $900 \times$ DHY 286-1 and G(B) 20 x G.Cot.10 registered maximum values of standard heterosis.

For number of seeds per boll, the standard heterosis varied from -24.51 to 32.65 per cent. Three crosses viz., G (B) 20 x DHY 286-1, G.Cot.100 x G.Cot.10 and G(B) 20 x G.Cot.10 showed significant and positive standard heterosis.

In seed index (g), the standard heterosis ranged between -13.27 to 28.95 per cent. Seven hybrids showed significant and positive standard heterosis, in which five crosses viz., G.Cot.100 x DHY 286-1, G(B) 20 x DHY 286-1, G(B) 20 x G.Cot.10, G.Cot.100 x G.Cot.10 and LH 900 x DHY 286-1 showed maximum values.

The heterosis over standard check ranged from -8.40 to 15.79 per cent in ginning percentage (\%). Three hybrids viz., PH 93 x G.Cot.10, PH 93 x DHY 286-1 and LRK 516 x G.Cot.10 exhibited significant and positive heterosis over standard check. For seed cotton yield per plant $(\mathrm{g})$, the heterosis over standard check ranged from -39.17 to 9.36 per cent. Only one hybrid $\quad \mathrm{G}(\mathrm{B}) \quad 20 \quad \mathrm{x} \quad$ G.Cot.10 exhibited significant and positive standard heterosis. The heterosis over standard check varied from -13.44 to -4.69 per cent in 2.5 per cent span length $(\mathrm{mm})$. None showed positively significant heterosis over standard check.

In fibre strength (g/tex), the standard heterosis varied from -11.44 to 8.36 per cent. Three hybrids viz., LH 900 x G.Cot.10, LH 900 x DHY 286-1 and LRA 5166 x DHY 286-1 exhibited significant and positive standard heterosis.

crosses viz., G (B) 20 x G.Cot.10, G(B) 20 x DHY-286-1 and LRK-516 $x$ DHY-286-1 performed better for standard heterosis, where their SCA effects were also significantly higher.

It was observed that hybrids showing high heterosis for seed cotton yield per plant in general also manifested heterotic effects for its contributing characters like number of monopodia per plant, number of sympodia per plant, number of bolls per plant, boll weight, number of seeds per boll and seed index. Similar results have been reported by Kajjidoni et al., (1999), Bhale and Bhat (1990), Srinivasan and Gururajan (1983), Tuteja et al., (2000), Tuteja and Singh (2001).

Singh and Murty (1971) reported heterosis to the extent of -76.2 to 137.2 per cent and -87.4 to 68.2 per cent over mid parent and better parent respectively in intra specific crosses of G.hirsutum L.

The maximum heterosis for yield in intra specific crosses was observed by Patel (1971). $\mathrm{He}$ reported 148 to 184 per cent heterobeltiosis for seed cotton yield in Hybrid4. Vadodaria and Patel (1995) reported high heterosis to the extent of -8.30 to 15.93 per cent and -23.94 to 112.09 per cent over better parent and standard check respectively in intraspecific crosses of G. hirsutum L. 
Table.1 Estimates of standard heterosis for different characters in cotton

\begin{tabular}{|c|c|c|c|c|c|c|c|c|c|c|c|c|}
\hline \multirow[b]{2}{*}{ Crosses } & \multicolumn{4}{|c|}{ Days to 50 per cent flowering } & \multicolumn{4}{|c|}{ plant height (cm) } & \multicolumn{4}{|c|}{ Monopodia per plant } \\
\hline & Loc-I & Loc-II & Loc-III & Pooled & Loc-I & Loc-II & Loc-III & Pooled & Loc-I & Loc-II & Loc-III & Pooled \\
\hline 76 IH 20 x G.Cot.10 & $-22.17 * *$ & $-16.02 * *$ & $-21.39 * *$ & $-19.80 * *$ & $19.46 * *$ & 5.52 & 6.43 & $10.48 * *$ & $48.85 * *$ & $56.68 * *$ & 19.58 & $41.27 * *$ \\
\hline 76 IH 20 x DHY 286-1 & $-14.29 * *$ & $-12.63 * *$ & -6.42 & $-11.24 * *$ & 7.86 & 11.09 & -4.73 & 4.96 & $-51.15 * *$ & 26.72 & -25.00 & $-16.87 *$ \\
\hline LH 900 x G.Cot.10 & -6.41 & $-20.88 * *$ & $-8.71 * *$ & $-15.27 * *$ & $-30.44 * *$ & $-31.54 * *$ & $-27.47 * *$ & $-29.87 * *$ & $51.15^{* *}$ & -13.77 & -2.92 & 12.45 \\
\hline LH $900 \times$ DHY 286-1 & -7.89 & $-17.97 * *$ & $-8.55^{*}$ & $-11.57 * *$ & $-16.51 * *$ & $-24.57 * *$ & $-33.70 * *$ & $-25.14 * *$ & -2.69 & $40.49 * *$ & 5.42 & 14.06 \\
\hline PH 93 x G.Cot.10 & $-28.08 * *$ & $-27.19 * *$ & $-32.09 * *$ & $-29.03 * *$ & 0.02 & 0.33 & 3.30 & -1.17 & $28.08^{*}$ & $37.65^{*}$ & 2.92 & $23.29 * *$ \\
\hline PH 93 x DHY 286-1 & $-14.29 * *$ & $-13.11 * *$ & $-18.71 * *$ & $-15.44 * *$ & 0.18 & $12.97 *$ & 0.58 & -4.74 & $-48.85 * *$ & -8.10 & -13.42 & $-29.32 * *$ \\
\hline LRA 5166 x G.Cot.10 & $-17.25 * *$ & $-27.19 * *$ & $-26.20 * *$ & $-23.49 * *$ & $-16.62 * *$ & $-23.34 * *$ & $-25.77 * *$ & $-21.87 * *$ & 23.08 & 13.36 & -22.08 & 5.22 \\
\hline LRA 5166 x DHY 286-1 & -4.94 & $-13.11 * *$ & -6.42 & $-8.22 * *$ & 0.44 & $-19.73 * *$ & $-11.17 *$ & $-10.26^{*}$ & $28.08^{*}$ & $32.39 *$ & $61.25 * *$ & $40.16^{* *}$ \\
\hline LRK 516 x G.Cot.10 & $-37.45 * *$ & $-33.01 * *$ & $-26.73 * *$ & $-32.55 * *$ & $-30.46 * *$ & $-33.21 * *$ & $-40.24 * *$ & $-34.54 * *$ & $-53.85 * *$ & 5.26 & 8.33 & $-14.46 * *$ \\
\hline LRK 516 x DHY 286-1 & $-18.72 * *$ & $-19.43 * *$ & $-19.78 * *$ & $-19.30 * *$ & $-34.38 * *$ & $-44.29 * *$ & $-20.65 * *$ & $-33.44 * *$ & $43.46 * *$ & 26.72 & $58.00 * *$ & $40.16^{* *}$ \\
\hline G(B) 20 x G.Cot. 10 & $-26.11 * *$ & $-24.28 * *$ & $-22.46 * *$ & $-24.33 * *$ & -7.54 & 7.72 & $18.14 * *$ & 5.94 & 20.38 & $70.04 * *$ & $44.58 * *$ & $44.58 * *$ \\
\hline G(B) 20 x DHY 286-1 & $-10.34 *$ & 0.00 & -5.87 & $-5.37 *$ & $-27.56 * *$ & -11.33 & 6.56 & $-11.05 * *$ & 0.00 & $53.85 * *$ & $83.33 * *$ & $44.58 * *$ \\
\hline G.Cot. 100 x G.Cot. 10 & -7.89 & $-9.71 *$ & 4.28 & $-4.70 *$ & 7.90 & 4.78 & -3.27 & 3.26 & $48.85 * *$ & 13.36 & $58.33 * *$ & $40.16^{* *}$ \\
\hline G.Cot.100 x DHY 286-1 & 5.91 & 3.39 & $9.63 * *$ & $6.20 * *$ & 4.73 & 5.58 & $-11.16^{*}$ & -0.05 & 20.38 & 8.10 & $63.75 * *$ & $30.12 * *$ \\
\hline S.E. \pm & 3.07 & 2.58 & 2.18 & 1.52 & 6.67 & 7.68 & 6.69 & 4.06 & 0.32 & 0.36 & 0.37 & 0.20 \\
\hline
\end{tabular}

Table. 2 Estimates of standard heterosis for different characters in cotton

\begin{tabular}{|c|c|c|c|c|c|c|c|c|c|c|c|c|}
\hline \multirow[b]{2}{*}{ Crosses } & \multicolumn{4}{|c|}{ Sympodia per plant } & \multicolumn{4}{|c|}{ Number of bolls per plant } & \multicolumn{4}{|c|}{ Boll weight (g) } \\
\hline & Loc-I & Loc-II & Loc-III & Pooled & Loc-I & Loc-II & Loc-III & Pooled & Loc-I & Loc-II & Loc-III & Pooled \\
\hline 76 IH 20 x G.Cot. 10 & -12.37 & -3.00 & $-33.93 * *$ & $-15.14 *$ & $-30.12 * *$ & $-29.54 * *$ & 5.70 & $-19.57 * *$ & 0.00 & 13.10 & $-22.30 * *$ & -2.58 \\
\hline 76 IH 20 x DHY 286-1 & $-37.07 * *$ & -0.53 & -3.15 & $-14.01 *$ & -7.07 & 3.16 & $22.41 * *$ & 5.19 & -7.29 & $22.02 * *$ & $-23.93 * *$ & -2.27 \\
\hline LH 900 x G.Cot.10 & 21.85 & $-40.28 * *$ & -14.37 & -11.22 & $-25.35 * *$ & -8.64 & $-32.84 * *$ & $-21.38 * *$ & 3.34 & 6.25 & $13.11 *$ & $7.32 *$ \\
\hline LH $900 \times$ DHY 286-1 & -9.18 & 15.91 & -3.51 & 1.62 & $-25.51 * *$ & $-12.69 *$ & -8.06 & $-15.76 * *$ & $12.46^{* *}$ & $40.18^{* *}$ & 1.97 & $18.76^{* *}$ \\
\hline PH 93 x G.Cot.10 & $-26.16^{*}$ & $-26.84 *$ & $-30.78 * *$ & $-27.75 * *$ & -6.61 & -4.04 & 10.85 & -0.64 & 0.00 & $13.39 *$ & $-24.26 * *$ & -2.89 \\
\hline PH 93 x DHY 286-1 & -20.13 & -7.93 & 18.51 & -4.59 & 11.06 & -3.46 & $44.23 * *$ & $15.29 * *$ & -7.29 & 11.90 & $-28.52 * *$ & $-7.53 *$ \\
\hline LRA 5166 x G.Cot.10 & 4.01 & 0.00 & $-44.05 * *$ & -11.22 & -0.16 & 4.60 & -29.53 & -6.88 & $13.98 * *$ & 7.44 & 12.46 & $11.34 * *$ \\
\hline LRA 5166 x DHY 286-1 & 16.94 & -11.51 & $-35.66^{* *}$ & -8.51 & -9.22 & 4.32 & $24.77 * *$ & 5.55 & $18.24 * *$ & -0.30 & $-19.34 * *$ & -0.10 \\
\hline LRK 516 x G.Cot.10 & -21.25 & 17.55 & -19.61 & -6.62 & $-16.43 * *$ & -1.02 & 0.19 & -5.98 & $13.89 * *$ & 13.10 & 9.51 & $6.39 *$ \\
\hline LRK 516 x DHY 286-1 & 13.79 & -2.47 & $28.32 *$ & 12.03 & 7.83 & 4.75 & -11.37 & 1.17 & $28.57 * *$ & 9.52 & $26.89 * *$ & $21.55^{* *}$ \\
\hline G(B) 20 x G.Cot. 10 & -13.23 & 0.29 & 12.59 & -0.90 & $23.34 * *$ & $27.94 * *$ & 5.51 & $19.90 * *$ & $12.77 * *$ & 13.10 & $14.10^{*}$ & $13.20 * *$ \\
\hline G(B) $20 \times$ DHY 286-1 & 23.84 & 12.33 & -18.20 & 7.61 & $-31.96 * *$ & -6.05 & $16.90 *$ & $-8.36^{*}$ & $30.40 * *$ & $44.35 * *$ & 7.54 & $28.04 * *$ \\
\hline G.Cot. 100 x G.Cot.10 & 8.92 & 0.00 & $-24.29 *$ & -3.92 & -4.31 & -8.64 & 4.60 & -3.33 & $28.57 * *$ & $22.02 * *$ & $39.34 * *$ & $29.59 * *$ \\
\hline G.Cot.100 x DHY 286-1 & -23.84 & $-36.99 * *$ & 16.41 & $-17.12 *$ & $23.50 * *$ & -1.45 & $19.46 *$ & $15.55^{* *}$ & 0.00 & $30.95 * *$ & 9.18 & $13.51 * *$ \\
\hline S.E. \pm & 2.76 & 2.81 & 2.15 & 1.50 & 2.29 & 2.75 & 2.88 & 1.53 & 0.12 & 0.22 & 0.19 & 0.10 \\
\hline
\end{tabular}


Table.3 Estimates of standard heterosis for different characters in cotton

\begin{tabular}{|c|c|c|c|c|c|c|c|c|c|c|c|c|}
\hline \multirow[b]{2}{*}{ Crosses } & \multicolumn{4}{|c|}{ Number of seeds per boll } & \multicolumn{4}{|c|}{ Seed index (g) } & \multicolumn{4}{|c|}{ Ginning percentage $(\%)$} \\
\hline & Loc-I & Loc-II & Loc-III & Pooled & Loc-I & Loc-II & Loc-III & Pooled & Loc-I & Loc-II & Loc-III & Pooled \\
\hline 76 IH 20 x G.Cot.10 & -12.25 & $-19.13 *$ & -22.53 & $-17.87 * *$ & -10.88 & -1.20 & $-29.73 * *$ & $-13.27 *$ & -7.52 & $-12.02 *$ & $12.47 * *$ & -2.34 \\
\hline 76 IH 20 x DHY 286-1 & -17.58 & -1.02 & 0.28 & -6.24 & 13.38 & -12.73 & 12.16 & 3.79 & -5.42 & -6.07 & 3.45 & -2.69 \\
\hline LH 900 x G.Cot. 10 & $-28.41 * *$ & -17.23 & $-28.20 *$ & $-24.51 * *$ & -8.75 & -1.20 & -15.27 & -8.09 & -2.98 & -7.93 & -11.09 & -7.30 \\
\hline LH 900 x DHY 286-1 & $-31.65 * *$ & $32.88 * *$ & -16.72 & -4.88 & 14.13 & 10.08 & 12.16 & $12.14^{*}$ & $-11.06^{*}$ & -9.66 & $10.31 *$ & -3.51 \\
\hline PH 93 x G.Cot.10 & $-19.22 *$ & $-33.14 * *$ & -12.61 & $-21.85 * *$ & -10.00 & 0.48 & 11.22 & 0.23 & $18.30 * *$ & $24.43 * *$ & 4.70 & $15.79 * *$ \\
\hline PH 93 x DHY 286-1 & -9.32 & -4.99 & -3.78 & -6.06 & 2.88 & -11.52 & -1.76 & -3.67 & $15.06 * *$ & $21.23 * *$ & 6.98 & $14.39 * *$ \\
\hline LRA 5166 x G.Cot.10 & -1.15 & -18.69 & -17.81 & $-12.44 *$ & 17.13 & -2.76 & $26.08 *$ & $12.90 *$ & 0.34 & $-18.63 * *$ & $18.80 * *$ & 0.31 \\
\hline LRA 5166 x DHY 286-1 & 12.61 & $-19.62 *$ & $-26.17 *$ & -10.76 & -9.62 & -0.72 & 8.51 & -0.88 & 5.36 & $14.32 *$ & -8.18 & 3.78 \\
\hline LRK 516 x G.Cot.10 & -17.75 & $-26.20 * *$ & 10.20 & -11.71 & 3.37 & -12.36 & $27.03^{*}$ & 5.18 & 9.39 & -5.95 & $23.94 * *$ & $9.26 * *$ \\
\hline LRK 516 x DHY 286-1 & 16.16 & -13.83 & 15.59 & 5.74 & 14.63 & -12.36 & $37.84 * *$ & $12.39 *$ & 0.40 & -7.81 & $17.66 * *$ & 3.49 \\
\hline G(B) 20 x G.Cot. 10 & $22.19 *$ & 9.46 & 3.21 & $11.76^{*}$ & 14.63 & $38.06 * *$ & 0.00 & $18.33 * *$ & -5.54 & -6.01 & $-13.34 * *$ & $-8.40 * *$ \\
\hline G(B) $20 \times$ DHY 286-1 & $29.29 * *$ & $35.09 * *$ & $33.68^{*}$ & $32.65 * *$ & 5.00 & $23.65^{*}$ & $50.95 * *$ & $25.92 * *$ & 7.40 & 9.48 & -8.76 & 2.70 \\
\hline G.Cot.100 x G.Cot.10 & 12.29 & -1.33 & $39.96 * *$ & $16.51 * *$ & 3.37 & 12.00 & $36.49 * *$ & $16.69 * *$ & $-13.36^{*}$ & -5.23 & $8.88 *$ & -3.33 \\
\hline G.Cot.100 x DHY 286-1 & $20.42 *$ & -3.09 & 14.93 & 10.67 & $27.88 * *$ & $33.25 * *$ & $25.27^{*}$ & $28.92 * *$ & -2.89 & 15.13 & 0.47 & 4.07 \\
\hline S.E. \pm & 2.01 & 2.14 & 2.69 & 1.32 & 0.80 & 0.86 & 0.81 & 0.47 & 1.89 & 1.90 & 1.50 & 1.02 \\
\hline
\end{tabular}

Table.4 Estimates of standard heterosis for different characters in cotton

\begin{tabular}{|c|c|c|c|c|c|c|c|c|c|c|c|c|}
\hline & \multicolumn{4}{|c|}{ Seed cotton yield per plant (g) } & \multicolumn{4}{|c|}{2.5 per cent span length $(\mathrm{mm})$} & \multicolumn{4}{|c|}{ Fibre strength (g/tex) } \\
\hline Crosses & Loc-I & Loc-II & Loc-III & Pooled & Loc-I & Loc-II & Loc-III & Pooled & Loc-I & Loc-II & Loc-III & Pooled \\
\hline 76 IH 20 x G.Cot. 10 & $-46.43 * *$ & $-35.52 * *$ & $-34.75^{* *}$ & $-39.17 * *$ & -5.74 & $-14.74 *$ & $-9.63 *$ & $-9.91 * *$ & 2.21 & 6.35 & 1.36 & 3.29 \\
\hline 76 IH 20 x DHY 286-1 & $-43.59 * *$ & -.094 & $-32.10 * *$ & $-23.92 * *$ & -4.53 & $-12.02 *$ & -23.80 & $-13.12 * *$ & -5.65 & 0.00 & -11.90 & -5.95 \\
\hline LH 900 x G.Cot.10 & $-52.08 * *$ & $-22.15 * *$ & $-43.37 * *$ & $-38.11 * *$ & -3.76 & -9.42 & -7.55 & $-6.83^{*}$ & 4.26 & 12.21 & 8.91 & $8.36 *$ \\
\hline LH 900 x DHY 286-1 & $-45.76^{* *}$ & -0.55 & $-31.21 * *$ & $-24.31 * *$ & $-9.90 *$ & 4.88 & $-10.78 *$ & -5.37 & 3.60 & 13.30 & 8.55 & $8.36^{*}$ \\
\hline PH 93 x G.Cot.10 & $-45.44 * *$ & $-13.95 * *$ & $-30.55^{* *}$ & $-29.28 * *$ & $-23.86 * *$ & $-13.49 *$ & $-26.18 * *$ & $-21.23 * *$ & -12.84 & -3.83 & $-17.30 *$ & $-11.44 * *$ \\
\hline PH 93 x DHY 286-1 & $-22.70 * *$ & $-17.08 * *$ & -5.65 & $-16.15^{* *}$ & $-14.42 * *$ & -5.57 & $-17.44 * *$ & $-12.51 * *$ & 4.26 & 13.30 & -1.78 & 5.14 \\
\hline LRA 5166 x G.Cot.10 & $-12.97 * *$ & $-10.83^{*}$ & $-32.54 * *$ & $-17.11 * *$ & -9.84 & $-15.95 * *$ & -4.24 & $-8.94 * *$ & -6.37 & 2.35 & -1.42 & -1.88 \\
\hline LRA 5166 x DHY 286-1 & $-24.24 * *$ & $-16.37 * *$ & $-15.69 * *$ & $-18.97 * *$ & $-14.93 * *$ & $-16.20 * *$ & -8.96 & -13.44 & 7.70 & $15.11 *$ & 0.68 & $7.73 *$ \\
\hline LRK 516 x G.Cot.10 & $-36.85 * *$ & $-12.82 * *$ & $-28.42 * *$ & $-25.27 * *$ & $-8.43^{*}$ & 0.04 & $-18.41 * *$ & $-8.87 * *$ & -9.09 & -0.77 & -12.43 & -7.53 \\
\hline LRK 516 x DHY 286-1 & 4.21 & $-13.40 * *$ & -2.90 & 4.51 & $-13.28 * *$ & -2.90 & -24.02 & $-13.34 * *$ & 1.03 & 10.56 & -3.88 & 2.45 \\
\hline G(B) 20 x G.Cot. 10 & $10.4^{*}$ & $20.46 * *$ & -9.13 & $9.36 * *$ & $-9.06 *$ & -4.44 & -10.30 & $-7.94 * *$ & -0.72 & 9.30 & 1.05 & 3.08 \\
\hline G(B) 20 x DHY 286-1 & $-32.27 * *$ & $13.01 * *$ & $19.12 * *$ & 1.41 & $-12.41 * *$ & -6.01 & -2.94 & $-7.30 * *$ & -3.44 & -1.86 & -0.73 & -1.99 \\
\hline G.Cot.100 x G.Cot.10 & -2.92 & $-14.73 * *$ & -8.81 & $9.06 * *$ & $-10.16^{*}$ & 3.67 & -7.14 & -4.69 & 0.51 & 9.09 & -0.89 & 2.82 \\
\hline G.Cot.100 x DHY 286-1 & 3.08 & $-14.08 * *$ & 8.93 & -2.17 & -7.31 & 9.66 & $-11.42 *$ & $-9.44 * *$ & 2.21 & 5.47 & -0.73 & 2.29 \\
\hline S.E. \pm & 6.50 & 7.01 & 5.75 & 3.72 & 1.21 & 1.54 & 1.22 & 0.77 & 1.24 & 1.30 & 1.27 & 0.73 \\
\hline
\end{tabular}


The moderate to high heterosis observed in present study has also been reported by several workers for number of bolls per plant (Desai et al., 1982; Duhoon et al., 1983; Tiwari et al., 1987; Kalsy and Garg 1989; Duhoon 1990; Patil et al., 1991; Siddique 1993 and Bhatade and Rajewar 1994) ; boll weight (Desai et al., 1982; Patil and Chopde 1985; Duhoon 1990; Patil et al., 1991and Bhatade et al., 1994); number of monopodia per plant (Khan and Ali 1980; Duhoon et al., 1983); number of sympodia per plant (Singh and Singh 1981; Duhoon et al., 1983); seed index (Singh and Singh 1981, Nadarajan and Sree Rangasamy 1990; Siddiqui 1993; Bhatade et al., 1994); ginning percentage (Singh and Singh 1981; Duhoon et al., 1983; Tiwari et al., 1987; Duhoon 1990, Gururajan and Basu 1992; Siddiqui 1993 and Bhatade and Rajewar 1994); early maturity (Patil and Sheriff 1980, Singh and Singh 1981, Patil and Chopde 1985, Siddiqui 1993, Vadodaria and Patel 1995); plant height (Singh and Singh 1981, Patil and Chopde 1985, Bhatade and Rajewar 1994); fibre length (Prakash 1982, Gururajan and Basu 1992, Duhoon et al., 1983, Duhoon 1990, Sidiqui 1993, Bhatade et al., 1994) and fibre strength (Prakash 1982).

Shroff et al., (1983) revealed that the inter specific hybrids produced by using cytoplasmic male sterility were at par in yield of seed cotton to the traditional hybrids i.e. Varalaxmi, JK Hy-1 and H 4. Srinivasan and Gururajan (1983) stated that the performance of reconstituted hybrids (Hybrid-4 and Varalaxmi developed by male sterility) was statistically on par with the conventional hybrids in respect of all economical and technical characters.

Prospects for successful production of pure and low cost first generation hybrid seed using CMS system appears to be bright in near future. These systems not only circumvent emasculation, but may even set aside the necessity of hand pollination by developing effective cross pollination system, possibly developing insect pollinators or at least by some mechanical device.

\section{References}

Khan, W.Cs. and Ali. Hidayat (1980). Hybrid vigour for seed cotton yield and its components in G. hirsutum L. crosses. Pakistan Cottons, 24 : 225-239.

Anonymous, (1993a). Annual report, CICR, Regional Station, Sirsa.

Anonymous, (1993b). Progress report, Marathwada Agri. Univ., Nanded.

Anonymous, (1993c). Progress report, CICR; Nagpur.

Bhale, N.L. and Bhat, M.G., (1990). Investigations on exploitation of heterosis in cotton ( $G$. hirsutum) using male sterility. Indian Journal of Genetics and Plant Breeding. 50 (1) : 37-44.

Bhatade, S.S. and Rajewar, S.R. (1994). Heterobeltiosis and standard heterosis for yield and quality characters in some $G$. hirsutum L. crosses. Madras Agric. J., 81 (1) : 34-35.

Bhatade, S.S.; Rajewar, S.R., Reddy, V.G. and Nadre, N.R. (1994). Phenotypic stability in cotton genotype of different species composition. J. Cott.Res. and dev., 8 (1) : 7-9.

Cook, C.G. and Namken, L.N. (1994). Performance of F1 hybrids in the lower Rio Grade Valley. Proceedings Beltwide cotton conferences, January 5-8, San Diego, CA, USA.

Desai, D.T.; Mehta, N.P. and Tikka, S.B.S. (1982). Heterosis for seed cotton yield, yield components and some fibre quality characters in upland cotton ( $G$. hirsutum L.). G.A.U., Res. J. 7 : 79-86.

Duhoon, S.S. (1990). Heterobeltiosis and exploitable heterosis for yield in a parental diallel crosses of American cotton (G. hirsutum L.). J. Indian Soc. 
Cott. Improv., 15 (2) : 80-87.

Duhoon, S.S.; Basu, A.K. and Sahni, V.M. (1983). Heterosis and combining ability studies in cotton ( $G$. hirsutum L.) $J$. Indian Soc. Cott. Improv.,8 (1) : 19-27.

Gunaseelan, T.; Basu, A.K. and Hanumantha Rao, H.K. (1996) Genetic evaluation of cytoplasmic male sterility based inter and intra specific hybrids in cotton. Journal of Indian Society for Cotton Improvement, $21: 28-32$.

Gururajan, K.N. and Basu, A.K. (1992). Heterosis and combining ability in medium staple cotton. J.Indian Soc. Cott. Improv.,17(1) :17-22.

Kajjiidoni, S.T.; Patil, S.J.; Khadi, B.M. and Salimath, P.M. (1999). A comparative study of heterosis in GMS based and conventional intra arboreum cotton hybrids. Indian J. Genet., 59 (4):493-504.

Kalsy, H.S. and Garg, H.R. (1989). Heterosis in intervarietal crosses of upland cotton (G. hirsutum L.). J. Indian Soc. Cott. Improv., 14 (2) : 159-162.

Khadi, B.M.; Kulkarni, V.N.; Katagari, I.S. and Soddi, R.R. (1998). Male sterility New Frontiers in Cotton Breeding. Proceedings of the world cotton research conference - 2, Anthens, Greece, September 6-12, 1998, pp. 246-249.

Li Yue You;Wang,X.D. and $\mathrm{Xu}$ YaNong.(2002). Cytological observations of cytoplasmic male sterile anther of cotton. Journal of Zhejiang University.(agriculture and life sciences), 28(1):11-15.

Moll, R.H.; Sathuana, W.S. and Robinson, H.F. (1962). Heterosis and genetic diversity in variety crosses of maize. Crop Sci., 2 : 197-198.

Nadarajan, N. and Sree Rangasamy, S.R. (1990). Study of heterosis and combining ability in G. hirsutum L. J. Indian Soc. Cott. Improv., 15(2) : 88-94.

Patel, C.T. (1971). "Hybrid-4" - a new hope towards self sufficiency in cotton in
India. Cott. Dev., (2) : 1-5.

Patil, F.B., Shinde, Y.M. and Thombre, M.V. (1991). Heterosis in multiple environments for yield components and its relation with genetic divergence in cotton. Indian J. Genet., 51(1) : 118-124.

Patil, M.S. and Sheriff, R.A. (1980). Diallel analysis of the inheritance of some quantitative characters in upland cotton (G. hirsutum L.). Mysore J. agric. Sci., $14: 287-294$.

Patil, R.A. and Chopde, P.R. (1985). Heterosis for yield and its traits in upland cotton. Indian J. agric. Sci., 55(6) : 402-406.

Prakash, R. (1982). Studies on heterosis effects on fibre characters in intraspecific crosses in American cotton. $(G$. hirsutum L.). J. Indian Soc. Cott. Improv., 7 (2) : 73-77.

Punitha, D. and Raveendran, T.S. (1999). Heterosis and combining ability studies in inter specific coloured linted upland cotton (G. hirsutum L.). Crop Research Hissar, 17 (2) : 245-249.

Raveendran, T.S.; Shanti, N.; Krishnadoss, D.; Selvaraj, U. and Narayanan, A. (1992). Development of new cyto-nuclear male sterile line in G. hirsutum L. cotton using $G$. harknessii Brandagee cytoplasm. Paper presented at the proceedings of the First Vasanthrao Naik Memorial National Seminar on Agricultural Sciences, Nagpur, India, Dec., 5-6, 1992.

Sheetz, R.H. (1985). General and specific combining ability in upland cotton $G$. hirsutum intra-specific crosses utilizing G. harknessii Brandagee male sterile cytoplasm. Diss. Abst. Int., 46 : 4208.

Shroff, V.N. (1985). Research review on work done at Indore on cytoplasmic male sterility in cotton. Paper presented at the proceedings of National Seminar on Rainfed Cotton Production Technology. Parbhani, Nagpur, India, May 24, 1985.

Shroff, V.N. ; Dubey, S.; Julka, R.; Pandey, S.C.; Jadhav, S.B. and Mandoli, K.C. 
(1983). Evaluation of commercial hybrids from cytoplasmic male

Siddiqui, M.A. (1993). Heterobeltiosis for seed cotton yield and related character Hirsutum cotton hybrids. J. Maharashtra agric. Univ., 18 (3) : 403-405.

Silva, F.P. da; Oliveira, J.F. Da; Alves, J.F. and Crisostomo, J.R. (1985). Heterosis, combining ability and gene action in cotton. Ravista Brasileira de Genetica,. 8 (2) : 303-318.

Singh, B.B. and Murty, B.R. (1971). Hybrid vigour in intervarietal crosses of upland cotton. Indian J. Genet., 31 : 1-7.

Singh, P. and Singh, H.G. (1981). Heterosis and inbreeding depression in upland cotton. Indian J. agric. Sci., 31 : 611-698.

Srinivasan, K. and Gururajan, K.N. (1983). Reconstitution of hybrid 4 and Varalaxmi cotton utilizing male sterile base. $J$. Indian Soc. Cott. Improv, 8 : 15-18.

Srinivasan, K. and Gururajan, K.N. (1983). Reconstitution of hybrid 4 and Varalaxmi cotton utilizing male sterile base. $J$. Indian Soc. Cott. Improv, 8 : 15-18.

Tiwari, V.N., Mandloi, K.C. and Rao, K. (1987). Heterosis and inbreeding depression in intraspecific crosses of upland cotton. Indian J. agric. Sci., 57 (5)
:313-17.

Tuteja, O.P. and Singh, D.P. (2001). Heterosis for yield and its components in Asiatic cotton hybrids based on GMS system under varied environments. Indian $J$. Genet., 61 (3) : 291-292.

Tuteja, O.P.; Singh, D.P; Narula, A.M. and Singh, U.V. (2000). Studies on heterosis for yield and quality characters over environments in desi cotton hybrids (GMS based). Cotton Improv., 25(1):2328.

Vadodaria, K.V. and Patel, R.H. (1995). Genetic analysis of qualitative and quantitative traits in upland cotton $(G$. hirsutum L.). Unpublished Ph.D. thesis submitted to G.A.U., S.K.Nagar.

Wang Xue De; Zhang, T.Z.; Pan Jia Jue.; Wang, X.D.; Zhang, T.Z; and Pan, J.J. (1997). Cyto plasmic effects of cytoplasmic male sterile upland cotton. Acta Agronomica Sinica, 23 (4) : 393399.

Zhu, X.F.; Wang, X.D.; Sun, J.; Zhang, T.Z. and Pan, J.J. (1998). Assessment of cytoplasmic effecests of cytoplasmic male sterile lines in upland cotton. Plant Breeding, 117 (6) : 549-552.

\section{How to cite this article:}

Shashibhushan, D. and Patel U. G. 2019. Heterosis studies in CMS based upland cotton (Gossypium hirsutum L.) hybrids. Int.J.Curr.Microbiol.App.Sci. 8(09): 1697-1704. doi: https://doi.org/10.20546/ijcmas.2019.809.192 